\title{
We need more powerful microsatellite assay for population genetic studies of Trichophyton rubrum
}

\author{
Pchelin IM, ${ }^{1 *}$ Kryuchkova MA, ${ }^{1}$ Bogdanova TV,${ }^{1}$ Chilina GA, ${ }^{1}$ Boronina LG, ${ }^{2}$ Olina ES, ${ }^{3}$ \\ Shurpitzkaya OA, ${ }^{1}$ Vasilyeva NV, ${ }^{1}$ Taraskina AE. ${ }^{1}$
}

${ }^{1}$ Kashkin Research Institute of Medical Mycology, North-Western State Medical University named after I.I. Mechnikov, St. Petersburg; 2 Ural State Medical University, Yekaterinburg; ${ }^{3}$ Sverdlovsk Regional Dermatovenereologic Dispensary, Yekaterinburg, Russia

\section{Objectives}

To study genetic structure of Russian $T$. rubrum population.

\section{Methods}

A total of $65 T$. rubrum clinical isolates were isolated in St. Petersburg and Yekaterinburg, Russia in the years 2015-2017. Species identification was performed by DNA sequencing of ITS region. Urease activity was assessed by use of Christensen's urea broth. We performed molecular strain typing by sequencing of TERG_02941 locus and amplification of eight microsatellite loci according to Gong et al. 2016 and two additional newly developed loci. Bruvo pairwise genetic distances were calculated on the basis of the lengths of microsatellite loci in polysat package for $R$ and further subjected to PCA analysis.

\section{Results}

The sequencing of ITS rDNA region revealed one genotype throughout the sample, identical to KT285224. All isolates were urease-negative. The protein-coding locus TERG_02941 was polymorphic at the position 793, containing alternatively adenine or guanine. For 65 isolates, we obtained 59 multilocus genotypes.

Fig. 2. Trichophyton rubrum. $N=65 . P C A$ analysis, based on Bruvo pairwise genetic distances.

Fig. 1. The number of distinct multilocus genotypes $\downarrow$ relatively to the number of microsatellite loci used.

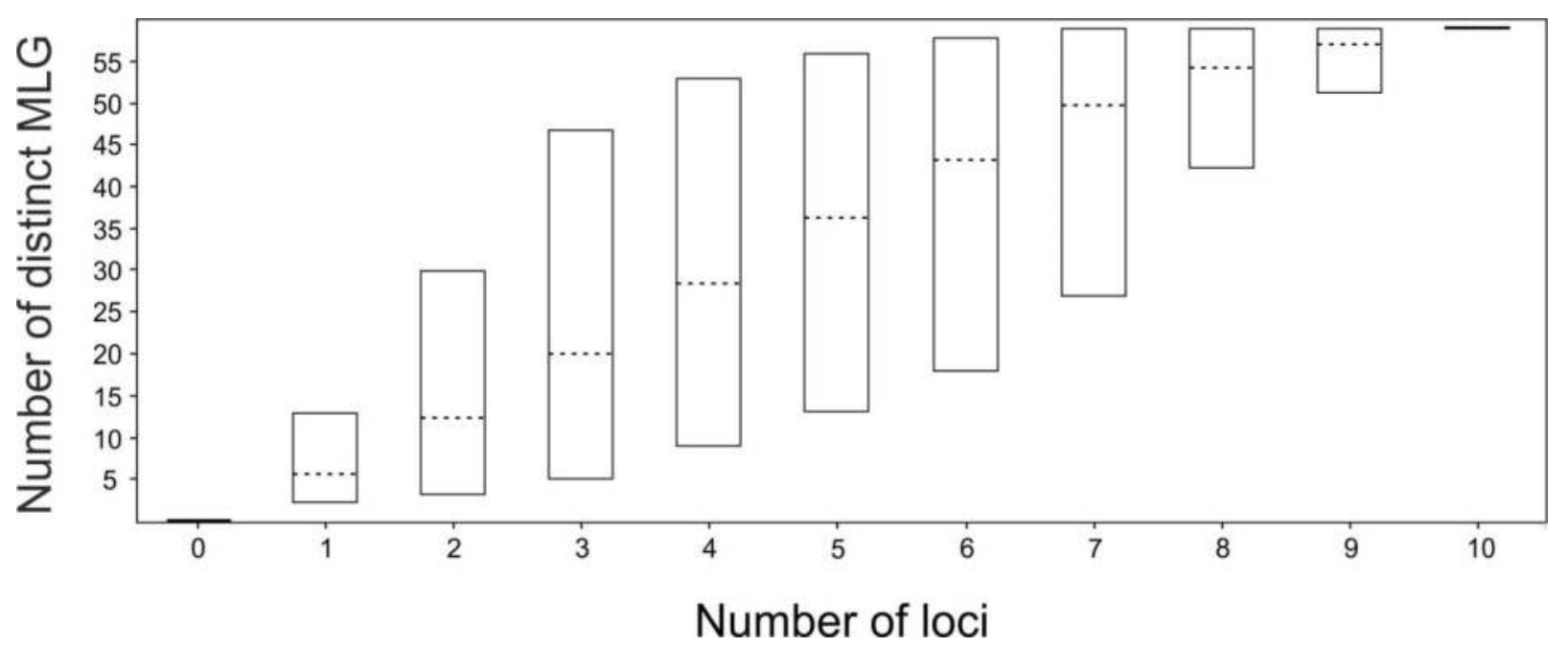

This corresponded to the genotypic richness $R$ value 0.91. Minimum combination of 7 loci Tr001, Tr002, Tr003, Tr004, Tr005, Tr006 and TrCT20 was sufficient for characterization of all obtained genotypes (Fig. 1). Shannon-Wiener index $\mathrm{H}^{\prime}$ was estimated as 4.038 (represents the uncertainty about the identity of an unknown individual), Shannon-Wiener eveness $\mathrm{V}^{\prime} \mathrm{H}^{\prime}$ was 0.990. The complement of Simpson's index, $D^{*}$ was 0.997 (represents the probability that two randomly chosen individuals belong to different genotypes). Eveness ED* was 0.791 . Principal component analysis of genetic distances contained two clouds, one for isolates with TERG_02941 genotype 793G, and another - mixed (Fig. 2). There was no correlation between body site and TERG_02941 genotype. Also, isolates from both cities of origin were interspersed.

\section{Conclusion}

It seems that Russian T. rubrum population consists from three subgroups. However, the discrimination power of available typing assays is not sufficient for clear delimitation of these subdivisions.

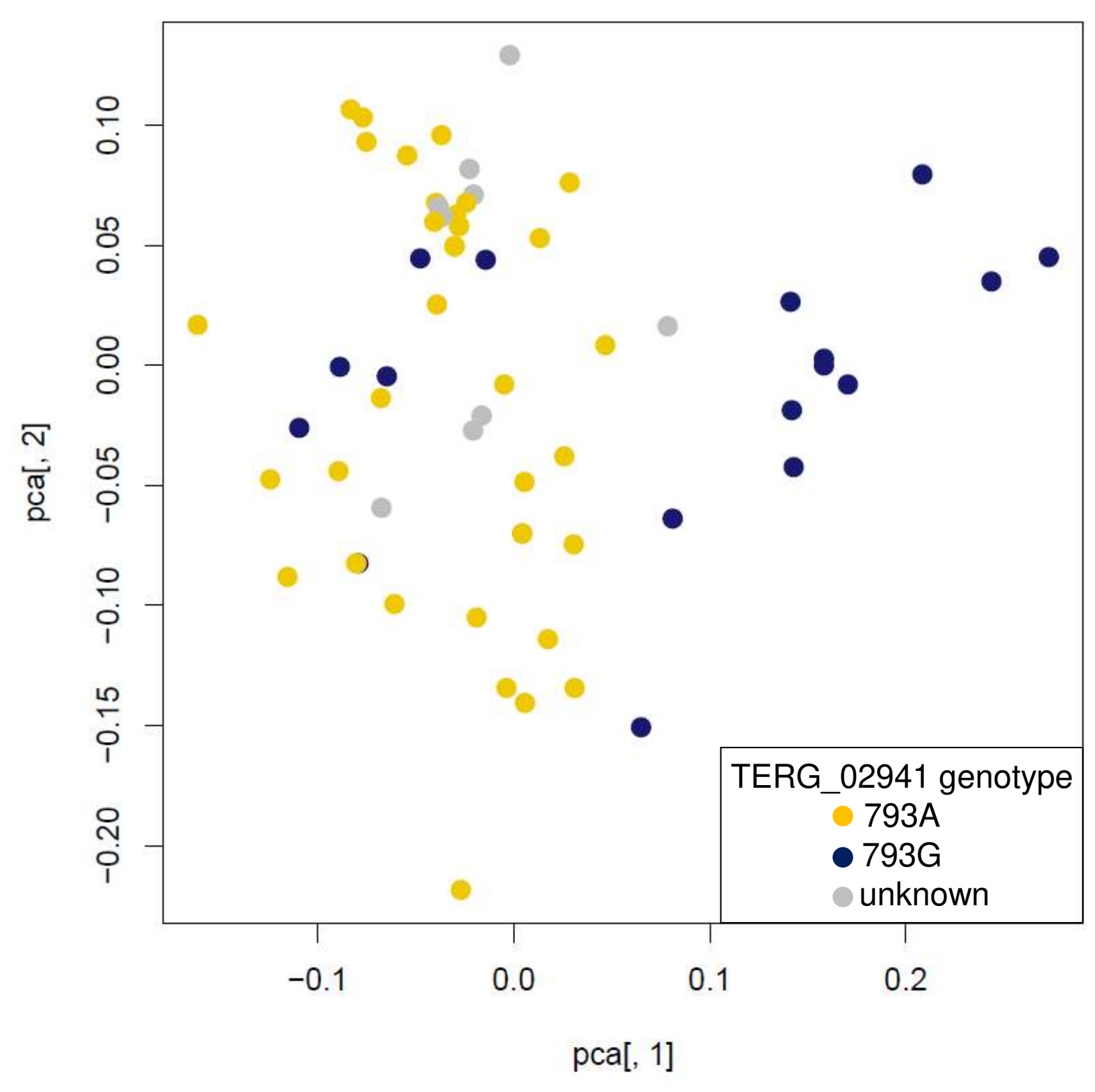

